\title{
Antimicrobial Susceptibility Pattern of Salmonella enterica Species in Blood Culture Isolates
}

\author{
Sunil Poudel ${ }^{1 *}$, Saroj Kumar Shrestha ${ }^{1}$, Ashish Pradhan ${ }^{1}$, Binaya Sapkota ${ }^{2}$ and Manoj Mahato ${ }^{1}$ \\ ${ }^{1}$ Department of Pathology, Civil Service Hospital, Minbhawan Kathmandu, Nepal \\ ${ }^{2}$ Department of Pharmacy, Civil Service Hospital, Minbhawan Kathmandu, Nepal
}

\begin{abstract}
Introduction: Enteric fever continues to be a major health problem in under developed countries including south Asian nations. In this study, we evaluate the prevalence and susceptibility pattern of Salmonella enteric (Serotype typhi, Paratyphi A and Paratyphi B).

Methods: Blood samples were obtained from 3210 patients, suspected with enteric fever. The sample was processed on BACTEC 9050 and isolates obtained from subculture were serotyped and antibiotic susceptibility testing was carried out using disk diffusion (Kirby-Bauer).

Result: Out of 3120 samples 370 isolates of S. enterica were isolated. The prevalence of Salmonella enterica was $11.8 \%$ where $78.4 \%$ of these isolates were S. enteric serotype Typhi, $20.8 \%$ were S. enteric serotype Paratyphi A and $0.8 \%$ were S. enteric serotype Paratyphi B. The isolates demonstrated poor susceptibility to oral antibiotics including Nalidixic acid, Ciprofloxacin, Ofloxacin, Azithromycin ,Amoxycillin, Tetracycline, Ceftriaxoneand Trimethoprim-sulfamethoxazole whereas all of the isolates of S. enterica demonstrated $100 \%$ susceptibility to Chloramphenicol.
\end{abstract}

Conclusion: There was grater prevalence of Salmonella enterica serotype typhi isolates resistant to fluoroquinolones (Nalidixic acid, Ofloxacin and Ciprofloxacin). However Chloramphenicol was sensitive to all isolates. This study suggests Chloramphenicol as a drug of choice for enteric fever and further monitoring of efficacy of older and newer antibiotics are desirable.

Keywords: Salmonela enterica, Blood culture, Multi Drug resistant, Antibiotics Susceptibility

\section{Introduction}

Enteric fever continues to be a major health problem in under developed countries including South Asian nations. It afflicts local inhabitants as well as travelers to endemic areas. Increasing multidrug resistance in Salmonella enterica serotype Typhi has been reported from various parts of the world [1-5]. Enteric fever is endemic in Nepal. S. enteric serotype Typhi and $S$. enteric serotype Paratyphi A have been reported as the most common culture isolates from patients with febrile illnesses needing hospital admission [6,7]. Over the past decade, increasing antibiotic resistance in $S$. enteric has led to a shift in the antibiotics used against this organism from chloramphenicol and ampicillin to trimethoprim-sulfamethoxazole, fluoroquinolones (ofloxacin, ciprofloxacin), and ceftriaxone. Even with the use of these antibiotics, the positive response to treatment has been only seen in the range of $16-40 \%$ in Nepal [7].

Over the last decade, fluoroquinolones have emerged as the mainstay of therapy for enteric fever. At the same time, increasing incidence of infection with Salmonellae resistant to nalidixic acid, which usually display decreased susceptibility to fluoroquinolones, has raised considerable global concern [8]. The vast majority of nalidixic acid resistant isolates remains within the current susceptibility range for ciprofloxacin $(1 \mu \mathrm{g} / \mathrm{ml})$ as recommended by the National Committee for Clinical Laboratory Standards (NCCLS). However, the probability of clinical response to fluroquinolone therapy in patients with invasive Salmonella infection is lower in those with Nalidixic acid resistant than with susceptible isolates [9]. In 1993, 23\% of Salmonella enteric serotype Typhi isolates from patients in the United Kingdom exhibited decreased susceptibility to ciprofloxacin more than half of these were also resistant to Chloramphenicol, Ampicillin and Trimethoprin. Increasing numbers of treatment failures were noted. Most infections were noted in patients with a recent history of travel to India and Pakistan, Nepal, Sri Lanka, Bangladesh, and Thailand [10].

\section{Materials and Methods}

The study was conducted from February to August 2013, at Civil Service Hospital at Kathmandu, Nepal. Blood samples were obtained from patients suspected with enteric fever. The samples to be tested were inoculated in Bactec ${ }^{\circ}$ culture bottle vial with soybean- casein digested broth which was inserted into the BACTEC $9050^{\circ}$ for incubation and periodic reading. Each culture vial contains a chemical sensor which can detect $\mathrm{Co}_{2}$ produced by the growth of microorganism. The sensor was monitored by the instrument every 10 minutes for an increase in its fluorescence, which was proportional to the amount of present. A positive reading indicated the presumptive presence of viable microorganism in the vial [11]. From the positive vial subculture was done on Blood agar and MacConkey agar, Identification of bacteria was done using standard microbiological techniques [12-14]. Serotyping of Salmonella enterica was done by done using polyvalent $\mathrm{O}$-antisera (Denka Seiken, Japan), Salmonella 9-O, Salmonella Vi(Remel Europe, UK). Antimicrobial susceptibility for Salmonella enterica was performed by Kirby- Bauer disk diffusion method on Mueller Hinton Agar following National Committee for Clinical Laboratory Standards (NCCLS) recommendations [12-15]. The antibiotics tested were: Amoxycillin $(10 \mu \mathrm{g})$ and Azithromycin $(15 \mu \mathrm{g})$ with zone of inhibition

*Corresponding author: Sunil Poudel, Department of Pathology, Civil Service Hospital, Minbhawan Kathmandu, Nepal, Tel:977-9841425162; E-mail: sooniil@gmail.com

Received January 29, 2014; Accepted April 08, 2014; Published April 18, 2014

Citation: Poudel S, Shrestha SK, Pradhan A, Sapkota B, Mahato M (2014) Antimicrobial Susceptibility Pattern of Salmonella enterica Species in Blood Culture Isolates. Clin Microbial 3: 141. doi:10.4172/2327-5073.1000141

Copyright: ( 2014 Poudel S, et al. This is an open-access article distributed under the terms of the Creative Commons Attribution License, which permits unrestricted use, distribution, and reproduction in any medium, provided the original author and source are credited. 
Citation: Poudel S, Shrestha SK, Pradhan A, Sapkota B, Mahato M (2014) Antimicrobial Susceptibility Pattern of Salmonella enterica Species in Blood Culture Isolates. Clin Microbial 3: 141. doi:10.4172/2327-5073.1000141

$(\mathrm{ZOI}) \geq 18 \mathrm{~mm}$, Ceftriaxone (30 $\mu \mathrm{g})$ and Ciprofloxacin $(5 \mu \mathrm{g}) \mathrm{ZOI} \geq 21$ $\mathrm{mm}$, Chloramphenicol $(30 \mu \mathrm{g}) \mathrm{ZOI} \geq 18 \mathrm{~mm}$, Co-trimoxazole $(25 \mu \mathrm{g})$ $\mathrm{ZOI} \geq \mathrm{mm}$, Nalidixic acid $(30 \mu \mathrm{g}) \mathrm{ZOI} \geq 19 \mathrm{~mm}$ and Tetracycline $(30$ $\mu \mathrm{g}) \mathrm{ZOI} \geq 15 \mathrm{~mm}$ (Hi Media Laboratory Ltd., Mumbai, India) [15]. The disk strength and zone-size interpretation were in accordance with the National Committee for Clinical Laboratory Standards (NCCLS) [15]. MDR was categorized if they were resistant to at least two classes of firstline agents including Amoxycillin, Chloramphenicol, Trimethoprimsulfamethoxazole, Fluoroquinolones (Ciprofloxacin and Ofloxacin), and Cephalosporins (Ceftriaxone).

Statistical comparisons of prevalence rates between the two serotypes and differences in resistance rates against the antibiotics were done by Fisher's exact tests and Chi-square tests and Fisher's exact test (where the sample size was less than 5) using SPSS software version 22.0 (SPSS Inc., Chicago, USA).

\section{Result}

Out of 3120 samples 370 isolates of S. enterica were isolated between
February to August 2013, at Civil Service Hospital Kathmandu. Among the blood cultures obtained from 3120 patients, $11.8 \%$ were positive for bacterial growth of S. enterica. Serotyping showed that 290 (78.4\%) of these isolates were S. enteric serotype Typhi, 77 isolates (20.8\%) were S. enteric serotype Paratyphi A and 3 isolates $(0.8 \%)$ were $S$. enteric serotype Paratyphi B. $(\mathrm{p}=0.000)$

Out of 370 isolates, (Table 1) 143 (38.6\%) isolates of Salmonella enterica serotype Typhi were sensitive to all antibiotics, $44(11.8 \%)$ isolates of Salmonella enterica serotype Paratyphi A and 2 (0.54\%) isolates Salmonella enterica serotype paratyphi B were sensitive to all antibiotics. Total 181 (48.91\%) isolates were resistant to two or more drugs.

Further, 35.5\% of MDR Salmonella enterica serotype typhi were combinely resistant to fluoroquinolones (Nalidixic acid, Ofloxacin and Ciprofloxacin) furthermore $18.7 \%$ isolates were resistant to the combination of Nalidixic acid and Ciprofloxacin whereas only $2.2 \%$ isolates were resistant to the combination of Nalidixic acid and Ofloxacin (Table 2).

\begin{tabular}{|c|c|c|c|c|c|c|c|c|c|c|}
\hline \multirow[t]{2}{*}{ Antibiotics } & \multicolumn{3}{|c|}{ Serotype typhi } & \multicolumn{3}{|c|}{ Serotype Paratyphi A } & \multicolumn{3}{|c|}{ Serotype Paratyphi B } & \multirow[t]{2}{*}{$P$ value } \\
\hline & $\mathbf{R}$ & I & $\mathbf{S}$ & $\mathbf{R}$ & I & $\mathbf{S}$ & $\mathbf{R}$ & I & $\mathbf{S}$ & \\
\hline Amoxycillin & 16 & 8 & 266 & 11 & 1 & 65 & 1 & 0 & 2 & 0.041 \\
\hline Azithromycin & 28 & 32 & 230 & 29 & 30 & 45 & 0 & 0 & 3 & 0 \\
\hline Ceftriaxone & 1 & 2 & 287 & 3 & 0 & 74 & 0 & 0 & 3 & 0.102 \\
\hline Ciprofloxacin & 122 & 49 & 119 & 3 & 20 & 54 & 0 & 1 & 2 & 0 \\
\hline Trimethoprim-sulfamethoxazole & 3 & 1 & 286 & 2 & 0 & 75 & 0 & 0 & 3 & 0.84 \\
\hline Chloramphenicol & 0 & 0 & 290 & 0 & 0 & 77 & 0 & 0 & 3 & - \\
\hline Ofloxacin & 87 & 33 & 170 & 2 & 0 & 75 & 0 & 0 & 3 & 0 \\
\hline Nalidixic_acid & 240 & 0 & 50 & 60 & 0 & 17 & 3 & 0 & 0 & 0.443 \\
\hline Tetracycline & 0 & 4 & 286 & 0 & 0 & 77 & 0 & 0 & 3 & 0.572 \\
\hline
\end{tabular}

S, susceptible; I, intermediately susceptible; R, resistant

Table 1: Antibiotic susceptibilities of Salmonella enteric serotype by Kirby-Bauer method

\begin{tabular}{|c|c|c|c|c|c|}
\hline S.N. & MultiDrug Resistant & Typhi & Paratyphi A & Paratyphi B & Total \\
\hline 1 & Non & 143 & 44 & 2 & 189 \\
\hline 2 & Azithromycin+Nalidixic acid & 13 & 22 & 0 & 35 \\
\hline 3 & Ciprofloxacin+Ofloxacin+Nalidixic acid & 68 & 0 & 0 & 68 \\
\hline 4 & Azithromycin+Ciprofloxacin+Ofloxacin+Nalidixic acid & 6 & 0 & 0 & 6 \\
\hline 5 & Amoxycillin+Nalidixic acid & 3 & 6 & 1 & 10 \\
\hline 6 & Amoxycillin+Azithromycin+Ceftriaxone+Nalidixic acid & 1 & 0 & 0 & 1 \\
\hline 7 & Amoxycillin+Azithromycin+Nalidixic acid & 0 & 1 & 0 & 1 \\
\hline 8 & Ofloxacin+Nalidixic acid & 4 & 0 & 0 & 4 \\
\hline 9 & Amoxycillin+Azithromycin+Ceftriaxone+Ciprofloxacin+Ofloxacin+Nalidixic acid & 0 & 1 & 0 & 1 \\
\hline 10 & Ciprofloxacin+Nalidixic acid & 34 & 0 & 0 & 34 \\
\hline 11 & Amoxycillin+Ceftriaxone+Ciprofloxacin+Trimethoprim+Nalidixic acid & 0 & 1 & 0 & 1 \\
\hline 12 & Amoxycillin+Ceftriaxone+Trimethoprim & 0 & 1 & 0 & 1 \\
\hline 13 & Trimethoprim+Ofloxacin+Nalidixic acid & 1 & 0 & 0 & 1 \\
\hline 14 & Azithromycin+Ciprofloxacin+Nalidixic acid & 1 & 0 & 0 & 1 \\
\hline 15 & Azithromycin+Ofloxacin+Nalidixic acid & 1 & 0 & 0 & 1 \\
\hline 16 & Amoxycillin+Ciprofloxacin+Nalidixic acid & 4 & 0 & 0 & 4 \\
\hline 17 & Amoxycillin+Ciprofloxacin+Ofloxacin+Nalidixic acid & 4 & 1 & 0 & 5 \\
\hline 18 & Azithromycin+Ciprofloxacin & 1 & 0 & 0 & 1 \\
\hline 19 & Ciprofloxacin+Ofloxacin & 3 & 0 & 0 & 3 \\
\hline 20 & Amoxycillin+Ofloxacin+Nalidixic acid & 1 & 0 & 0 & 1 \\
\hline 21 & Amoxycillin+Ciprofloxacin+Trimethoprim+Ofloxacin+Nalidixic acid & 1 & 0 & 0 & 1 \\
\hline \multirow[t]{2}{*}{22} & Trimethoprim+Nalidixic acid & 1 & 0 & 0 & 1 \\
\hline & Total & 290 & 77 & 3 & 370 \\
\hline
\end{tabular}

Pearson Chi-Square $=109.564, \mathrm{P}$ value $=0.000$

Table 2: Sus0063eptibilities of MDR Salmonella enteric serotype. 
Citation: Poudel S, Shrestha SK, Pradhan A, Sapkota B, Mahato M (2014) Antimicrobial Susceptibility Pattern of Salmonella enterica Species in Blood Culture Isolates. Clin Microbial 3: 141. doi:10.4172/2327-5073.1000141

In the other hand $7.18 \%$ of Salmonella enterica serotype typhi isolates were resistant to Azithromycin and Nalidixic acid whereas in case of Salmonella enterica serotype paratyphi A, the resistant pattern has increased up to $11.7 \%$.

\section{Discussion}

The result of this study showed that a prevalence rate of $11.8 \%$ for the $S$. enteric in the study area, a similar trend has been reported in a recent study done in Nepal [15]. Chloramphenicol, Ampicillin, and Trimethoprim-sulfamethoxazole were the antibiotics widely used as the primary for the treatment of treatment of enteric fever. In our study $48 \%$ of isolates were resistant to two or more drugs where $81.2 \%$ were S. enteric serotypes Typhi and $18.2 \%$ were Paratyphi A and only $0.5 \%$ were Paratyphi B (Table 3 ). All of these multidrug resistant isolates showed reduced susceptibility to fluoroquinolones (Table 2). A similarresults show a high sensitivity of both Salmonella enterica serovar typhi (96\%) and Salmonella enterica serovar paratyphi A (100\%) to chloramphenicol. Sensitivity to ciprofloxacin and amikacin was $88 \%$ and $84 \%$ respectively (Table 1). Sensitivity of Salmonella enterica serovar paratyphi A was $100 \%$ to Chloramphenicol, Ciprofloxacin, Ofloxacin, Nalidixic acid and Ceftriaxone, $95 \%$ to Amikacin and 30\% to ampicillin [16]. Our result (Table 1 and 4) showed different comparable view (i.e. Chloramphenicol $100 \%$ susceptibility) than that of others studies reporting Chloramphenicol resistant in S.enterica [17-19]. In 1970s the isolates resistant to Chloramphenicol were reported in various countries like UK, India, Mexico, Greece, Israel, and Pakistan [2,4]. While In the 1990s, the patter showing resistance of Chloramphenicol suggested to the use of fluoroquinolones (Ciprofloxacin and Ofloxacin) [20], which werefound to be highly resistant in this study which accounts as $40 \%$. Similarly a different view was reported on Pakistan reporting Salmonella enteric serotype paratyphi-A were sensitive to Ciprofloxacin, Ofloxacin and Nalidixic acid and $62.7 \%$ isolates were resistant to firstline antibiotics (Ampicillin, Chloramphenicol and Co-Trimoxazole) on disc diffusion testing[21]. According to Thonget et al. [5] The MDR S. typhi isolates were resistant to ampicillin, chloramphenicol, and trimethoprim-sulfamethoxazole. Analysis by PFGE showed that 50 MDR isolates of S. typhi had a single, homogenous PFGE profile, which was distinctly different from that of 50 antibiotic-sensitive isolates obtained in the same time frame from the same area [22].

\begin{tabular}{|l|c|c|c|c|}
\hline Organism & N & Percent & Chi-Square & P Value \\
\hline Salmonella enterica serotype Typhi & 290 & 78.37 & \multirow{2}{*}{360.038} & \multirow{2}{*}{0} \\
\cline { 1 - 3 } Salmonella enterica serotype Paratyphi A & 77 & 20.81 & & \\
\cline { 1 - 3 } Salmonella enterica serotype Paratyphi B & 3 & 0.81 & & \\
\cline { 1 - 3 } Total & 370 & 100 & & \\
\hline
\end{tabular}

Table 3: Frequency of Salmonella enteric serotypes.

\begin{tabular}{|l|c|c|c|}
\hline Antibiotic & Resistant & Intermidate & Sensitive \\
\hline Amoxycillin & $28(7.6 \%)$ & $9(2.4 \%)$ & $333(90 \%)$ \\
\hline Azithromycin & $57(15.4 \%)$ & $35(9.5 \%)$ & $278(75.1 \%)$ \\
\hline Ceftriaxone & $4(1.1 \%)$ & $2(0.5 \%)$ & $364(98.4 \%)$ \\
\hline Ciprofloxacin & $125(33.8 \%)$ & $70(18.9 \%)$ & $175(47.3 \%)$ \\
\hline Trimethoprim-sulfamethoxazole & $5(1.4 \%)$ & $1(0.3 \%)$ & $364(98.4 \%)$ \\
\hline Chloramphenicol & 0 & 0 & $370(100 \%)$ \\
\hline Ofloxacin & $89(24.1 \%)$ & $33(8.9 \%)$ & $248(67 \%)$ \\
\hline Nalidixic_acid & $303(81.9 \%)$ & 0 & $67(18.1 \%)$ \\
\hline Tetracycline & 0 & $4(1.1 \%)$ & $366(98.9 \%)$ \\
\hline
\end{tabular}

Table 4: Antibiotic susceptibility.

\section{Conclusion}

Our study has further accentuated concern about the sensitivity pattern and the status of multi drug resistant Salmonella enterica spp. This study suggests Chloramphenicol as a drug of choice for enteric fever and further monitoring of efficacy of older and newer antibiotics are desirable.

\section{Authors' Contributions}

Sunil Poudel: Sample collection, Identification and Antibiotic Susceptibility Testing, Monitoring and Evaluation, Literature Review, Data analysis, Manuscript preparation and Submission.

Saroj Kumar Shrestha: Identification and Antibiotic Susceptibility Testing Monitoring, Evaluation, Literature and Review

Ashish Pradhan: Sample collection, Identification and Antibiotic Susceptibility Testing, Monitoring and Evaluation, Literature Review and Manuscript preparation

Dr. Binaya Sapkota: Literature Review, Data analysis and Manuscript preparation

Manoj Mahato: Sample collection, Identification and Literature review

All authors read and approved the final manuscript.

\section{Acknowledgement}

We would like to acknowledge to all of the patients involved in this study. No funding was received for this research in its present form.

\section{References}

1. Pokharel BM, Koirala J, Dahal RK, Mishra SK, Khadga PK, et al. (2006) Multidrug-resistant and extended- spectrum beta-lactamase (ESBL)- producing Salmonella enteric (serotype Typhi and Paratyphi A) from blood isolates in Nepal: surveillance of ressistance and a search for newer alternatives. International Journal of Infectious Disease10: 434-438.

2. Rowe B, Ward LR, Threlfall EJ (1997) Multidrug-resistant Salmonella typhi: a worldwide epidemic. Clin Infect Dis 24 Suppl 1: S106-109.

3. Le TA, Lejay-Collin M, Grimont PA, Hoang TL, Nguyen TV, Grimont F, et al (2004) Endemic, epidemic clone of Salmonella entericaserovarTyphiharboring a single multidrug-resistant plasmid in Vietnam between 1995 and 2002. J ClinMicrobiol42: 3094-3099.

4. Gautam V, Gupta NK, Chaudhary U, Arora DR (2002) Sensitivity pattern of Salmonella serotypes in Northern India. Braz J Infect Dis 6: 281-287.

5. Thong KL, Bhutta ZA, Pang T (2000) Multidrug-resistant strains of Salmonella enterica serotype Typhi are genetically homogenous and coexist with antibioticsensitive strains as distinct, independent clones. Int J Infect Dis 4: 194-197.

6. Murdoch DR1, Woods CW, Zimmerman MD, Dull PM, Belbase RH, et al (2004) The etiology of febrile illness in adults presenting to Patan hospital in Kathmandu, Nepal. Am J Trop Med Hyg 70: 670-675.

7. Biswas R, Dhakal B, Das RN, Shetty KJ (2004) Resolving diagnostic uncertainty in initially poorly localizable fevers: a prospective study. Int J ClinPract 58: 2628.

8. Aarestrup FM, Wiuff C, Mølbak K, Threlfall EJ (2003) Is it time to change fluoroquinolone breakpoints for Salmonella spp.? Antimicrob Agents Chemother 47: 827-829.

9. Threlfall EJ, Ward LR, Skinner JA, Smith HR, Lacey S (1999) Ciprofloxacinresistant Salmonella typhi and treatment failure. Lancet 353: 1590-1591.

10. Threlfall EJ, Ward LR (2001) Decreased susceptibility to ciprofloxacin in Salmonella enterica serotype typhi, United Kingdom. Emerg Infect Dis 7: 448450

11. BD BACTECTM standard/ 10 Aerobic/ F culture vials, Cat. No.442260 442192,442193, Becton, Dickinson and Company, Sparks USA.

12. Collee JG, Dugoid JP, Fraser AG, Marmion BP. Mackie and McCartney Practical Microbiology 13th ed. Edinburgh, UK: Churchill Livingstone 1989.

13. Cheesbrough M. Medical Microbiology for Tropical Countries Vol. II. Microbiology 1 ELBS ed. Cambridge, UK: University Press; 1984;

14. Forbes BA, Sahm DF, Weissfield AS. Bailey \& Scott's Diagnostic Microbiology $11^{\text {th }}$ ed. St. Louis, USA: Mosby; 2002;p. 36 
Citation: Poudel S, Shrestha SK, Pradhan A, Sapkota B, Mahato M (2014) Antimicrobial Susceptibility Pattern of Salmonella enterica Species in Blood Culture Isolates. Clin Microbial 3: 141. doi:10.4172/2327-5073.1000141

15. Wikler MA, Cockerill FR, Craig WA, Dudley MN, Eliopoulos GM. National Committee for Clinical Laboratory Standards. Performance standards for antimicrobial susceptibility testing. Document M100-S14. Wayne, PA: NCCLS 2004. p. $30-5$

16. Bhatia JK, Mathur AD, Arora MM (2007) Reemergence of chloramphenicol sensitivity in enteric fever. Medical Journal Armed Forces India 7: 212-214.

17. Bhatta CP, Bhuyan KC, and Maharjan A. (2005) The study, antibiotic sensitivity pattern ofSalmonella species isolated from blood culture. Journal of Nepa Health Research Council; 3(2).

18. Verghese SL, Manonmani R, Balasubramanian S, Chandrasekharan S (1992) Multi-drug resistance in salmonellae isolated from enteric fever cases at Porur-a semi urban area near Madras City. J Commun Dis 24: 12-15.
19. Maheshwari VD, Agarwal SK (1996) Present status of drug resistance in cases of enteric fever in Rajasthan. J Assoc Physicians India 44: 618-619.

20. Prabhakar H, Kaur H, Lal M (1996) Prevalence of multi-drug resistant Salmonella typhi in Ludhiana Punjab. Indian J Med Sci 50: 277-279.

21. Parry CM, Hien TT, Dougan G, White NJ, Farrar JJ (2002) Typhoid fever. N Engl J Med 347: 1770-1782.

22. ParveenA, Qureshi AH, Muhammad SP, Muhammad ZUH, Mamoona H Increasing prevalence of multidrug resistant Salmonella enterica serotype paratyphi-A in patients with enteric fever. Pakistan J Med Res 2004;43:2. 